\title{
Cross Validation Analysis of Convolutional Neural Network Variants with Various White Blood Cells Datasets for the Classification Task
}

\author{
https://doi.org/10.3991/ijoe.v18i02.27321 \\ Syadia Nabilah Mohd Safuan ${ }^{1}$, Mohd Razali Md Tomari ${ }^{1,2(\bowtie),}$ \\ Wan Nurshazwani Wan Zakaria ${ }^{1}$ \\ ${ }^{1}$ Faculty of Electronic and Electrical Engineering, Universiti Tun Hussein Onn Malaysia, \\ Batu Pahat, Malaysia \\ ${ }^{2}$ Research Centre for Applied Electromagnetics, Johor, Malaysia \\ mdrazalieuthm.edu.my
}

\begin{abstract}
White Blood Cells (WBCs) analysis is an important procedure to detect diseases is that closely related to human immunity system. Manual WBCs analysis is laborious and hence computer aided system (CAD) is a better option to alleviate the shortcoming. Since conventional segmentationclassification approach is tedious to configure, a Convolutional Neural Network $(\mathrm{CNN})$ become recent trend for WBCs classification. Previously, there are many works proposed for WBCs identification. However, the models that can be generalised to works well among various datasets is remain vague. In this paper, an analysis of various $\mathrm{CNN}$ models which are simple Alexnet, embedded friendly Mobilenet, inception based Googlenet, systematic architecture VGG16 and skip connection based model (Resnet \& Densenet), are tested with three major WBCs datasets (Kaggle, LISC and IDB-2). From the rigorous experiments, it can be concluded that simple CNN model of Alexnet performs well across all three datasets with $98.08 \%$ accuracy on Kaggle, $96.34 \%$ accuracy on IDB-2 and $84.52 \%$ on LISC. This outcome can be utilise as a basis to improve the $\mathrm{CNN}$ classification model that can be generalize to works under various WBCs datasets.
\end{abstract}

Keywords - computer aided system, convolutional neural network, white blood cell

\section{Introduction}

White Blood Cell (WBC) is one of the important elements in human's body including Red Blood Cell (RBC) and platelets. It is greatly related to human's immune system that keeps the body healthy. Other than that, it helps to fight viruses and bacteria which will help to prevent any serious disease from attacking. However, an abnormal count of WBC could be harmful as it will also lead to several diseases such as Leukemia, Cancerous and other blood related diseases [1]. This is where WBC analysis is important so that early prevention and be made and the risk can be reduced. Other 
than that, WBC analysis also can help in monitoring the patient's health condition and the effectiveness of cancer patient treatment.

There are 5 types of WBC which are Eosinophil, Neutrophil, Basophil, Lymphocyte and Monocyte [2]. These cells are different with each other in terms of their shapes, number of lobes and sizes of its nucleus and cytoplasm [3]. The differences can be seen in Figure 1 and their nucleus' stain is also different [4]. As can be seen, the shape of Eosinophil and Neutrophil is more rounded compared to Monocyte that shows the irregular edges. Other than that, the cytoplasm's color resolution is also different form Monocyte, Lymphocyte and Basophil which is in deeper purple colored stain while Eosinophil and Neutrophil is more pinkish and light in color. The number of nucleus and its shape also can be observed to differentiate between these cells. Other than that, there are small cavities in the cytoplasm which is called vacuoles, seen in Eosinophil, Neutrophil and Basophil. The normality and number of each cell type must be monitored to ensure the patient is in a healthy condition.

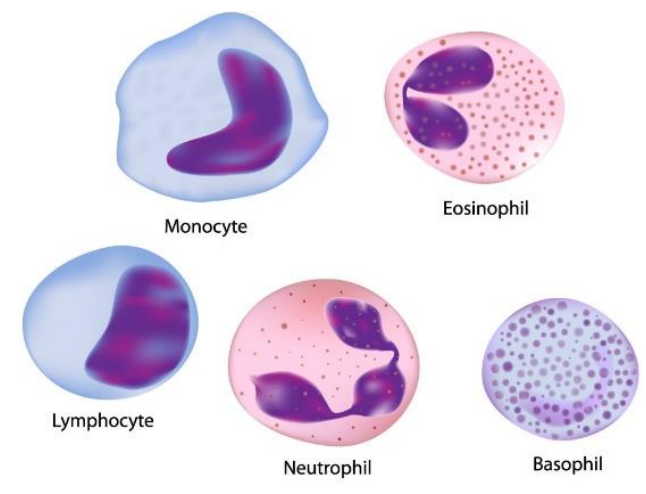

Fig. 1. 5 types of WBC

Traditionally, the WBC is done manually by the pathologist and it is very time consuming. Other than that, it is also challenging for the pathologist as the sample increases [5] and it is highly dependent on the pathologist's skill which will be confusing and yield inaccurate result [2]. However, industry has come out with a hematology machine that is automated, fast and accurate but these machines are really expensive and it is not portable [6]. Other than that, some researchers have been classifying WBC using conventional method that includes processes such as preprocessing, segmentation, feature extraction. The main problem with conventional method is that blood smear images can be affected by different conditions, light distribution and variation of staining intensities which can influence the segmentation process and can reduce the rate of segmentation [7].

As mentioned, these are the reasons that motivate this paper to classify WBC types using deep learning techniques which is Convolutional Neural Network (CNN). One of the advantages is deep learning can learn and extract high level features automatically and perform classification in the same time [8]. Features extraction is critically important as the wrong features selection will reduce the classification accuracy. 
Other than that, it is less complex without much tuning needed and less complicated process compared to the conventional method [9]. CNN is specifically developed to tackle feature extraction issue including variation of image rotation.

CNN basically automatically learn the features through backpropagation by using multiple building blocks, such as convolution layers, pooling layers, and fully connected layer [10]. CNN has proven an impressive performance in various tasks such as image classification, object detection, action recognition and many more since last few years [11]. Its ability to be both translation and rotation invariant has helped a lot of researchers to complete various tasks [12]. Deep learning is also widely used for brain tumour classification [13, 14], Korean character recognition [15] and leaves classification for Chinese herbal medicine [16]. Other than that, there are also research on offline signature verification using deep learning CNN [17]. Some works used googlenet from $\mathrm{CNN}$ for lung cancer detection $[18,19]$. However, this paper is focused on image classification of 5 WBC types as CNN works wonderfully and able to provide high performance on image classification [20]. There are many researches on WBC classification using CNN but most of it is only limited to one dataset and not much comparison has been made.

Some previous works that used CNN to classify WBC including classification that is based on Regional Convolutional Neural Network (R-CNN) [1]. There are four models used to train which are Alexnet, VGG-16, Googlenet and Resnet50 and Resnet50 is able to show the highest performance which is $100 \%$ and testing result of 99.52\%, 98.40\%, 98.48\%, 96.16\% and 95.04\% for Lymphocyte, Monocyte, Basophil, Eosinophil and Neutrophil respectively. Other than that, [7] and [8] develops their own model and the average accuracy achieved is $98.61 \%$ and $96.60 \%$ respectively. Next, WBC classification and counting is done by [2] which Alexnet has outperform Googlenet and Resnet-101. Other than that, five layers CNN model which contains three layers for feature extraction and the other two layers are used for classification [21]. Lastly, WBC detection and identification using modified LeNet-5 is proposed in [6].

Basically, this paper discusses about WBC types classification using deep learning which is CNN. The comparison between several pre-trained models which are Alexnet, Googlenet, VGG-16, Mobilenet, Resnet and Densenet is made to classify WBC types in blood smear image. The result of training and testing performance is compared to each other. Other than that, it is also tested on three different datasets which are Kaggle, IDB-2 and LISC to prove that the model is optimal and valid for most of WBC dataset. Matlab R2020a is the platform used with deep network designer toolbox.

\section{System overview}

\subsection{Platform}

The platform used in this paper is Matlab R2020a as it is a high level computer programming language that can calculate, represent, visualize and complete many 
other tasks. The main toolboxes used are deep learning, image processing and computer vision. In order to use the deep learning designer toolbox is to import the pretrained model and modify accordingly. After the modification is done, the model is exported to the Matlab workspace and executed for training process.

\subsection{Datasets}

There are three databases experimented in this project which are Kaggle, IDB-2 and LISC. These databases consist images of various types of WBC.

\section{Kaggle}

There are four types of WBC in Kaggle dataset which are Eosinophil, Neutrophil, Lymphocyte and Monocyte. Each class contains different number of images. As for Eosinophil, there are 2497 images, 2499 images of Neutrophil, 2483 images of Lymphocyte and 2478 images of Monocyte. This will total up to 9957 images in Kaggle dataset for training. The sample images in Kaggle can be seen in Figure 2. All the images are in RGB and in various rotation. The pinkish colored stain represents the Red Blood Cell (RBC) while the bright purple colored stain represents the WBC. There are various image rotation and augmentation in this dataset. A higher variance of data augmentation is needed to prevent model overfitting.

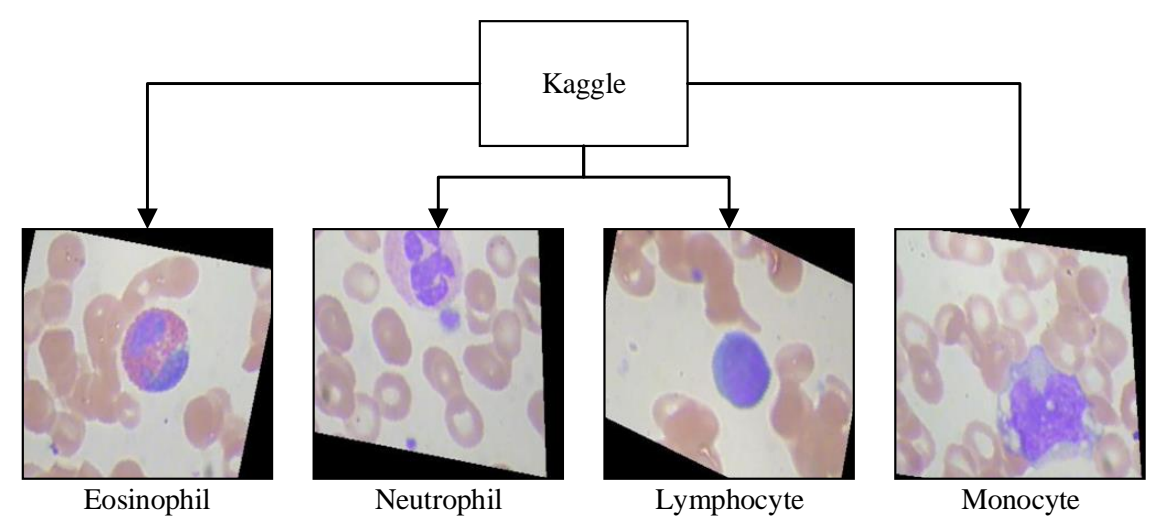

Fig. 2. Sample images in Kaggle database

\section{IDB-2}

IDB-2 database is used for Acute Lymphoblastic Leukemia (ALL) detection by classifying normal Lymphocyte and abnormal Lymphocyte that is called Lymphoblast. Basically, there are two classes of data which are Lymphoblast and NonLymphoblast. Figure 3 shows the sample images in IDB-2 database. The first row is the Lymphoblast cell and the second row is Non-lymphoblast cell. Each class contains 130 images, and the total images in IDB-2 dataset is 260 images. As seen in the figure, the pinkish brown is the $\mathrm{RBC}$, the deep purple is nucleus of the WBC and the 
light purple colored stain or the outer layer is the cytoplasm of the WBC. The differences between lymphoblast and non-lymphoblast cell is its shape irregularities, the size of the nucleus, the area of the cytoplasm and number of lobes in the nucleus.

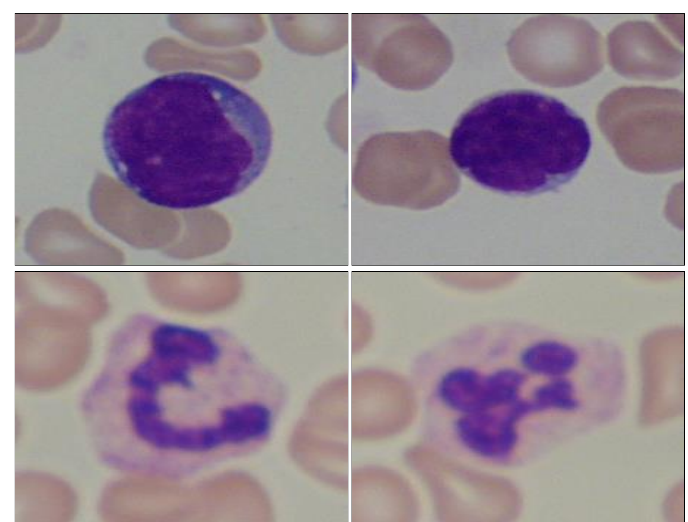

Fig. 3. Sample images in IDB-2 database

\section{LISC}

This dataset contains full set of WBC types which are Eosinophil, Neutrophil, Basophil, Monocyte and Lymphocyte which consist of 39, 50, 53, 52 and 48 respectively. Total image in this dataset is 242 images. This dataset is different from IDB and Kaggle dataset as the image magnification is different as depicted in Figure 4. In this dataset, there are more appearance of noises such as Red Blood Cell (RBC) and platelets. The bright colored region represents the WBC and the other small particles are RBC and platelets. There are also color variation in this dataset that makes it challenging to classify the cells. 


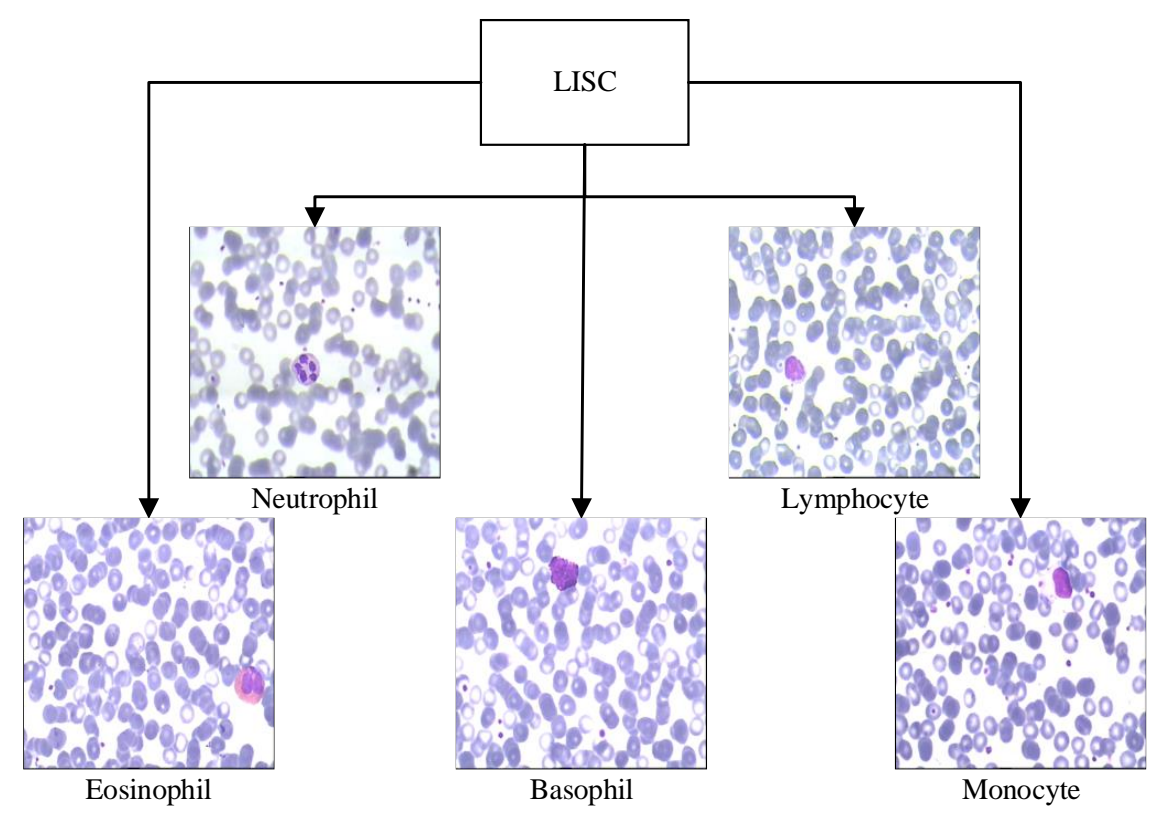

Fig. 4. Sample images in LISC dataset

\subsection{CNN models}

There are several main keys operation in $\mathrm{CNN}$ which include convolution, pooling and fully connected. Basically, convolution contains set of filters to obtain the feature maps and to learn feature representation of the inputs. Next, pooling is a down sampling layer to reduce the feature dimensions which will prevent overfitting. It is also equivalent to fuzzy filtering which is used to increase the robustness of feature extraction. Lastly, fully connected layer that acts as classifier which computes the last score. There are six models involved in this experiment which are Alexnet, Googlenet, VGG-16, Resnet, Mobilenet and Densenet. These models are tested because of its special feature such as the number of layers, present of inception module, skip connection, depthwise convolution and dense block. The batch size and epoch is fixed to 64 and 50 respectively.

\section{Alexnet}

Alexnet contains 8 layers of convolutional layer and fully connected layer as shown in Figure 5. The model also includes local response normalization and max pooling layer before fully connected layer. Input image must be in the size of $227 \times 227$ image resolution. First convolution mask of $11 \times 11$ is applied on the input image and followed by $5 \times 5$ and three times of $3 \times 3$ convolution. 


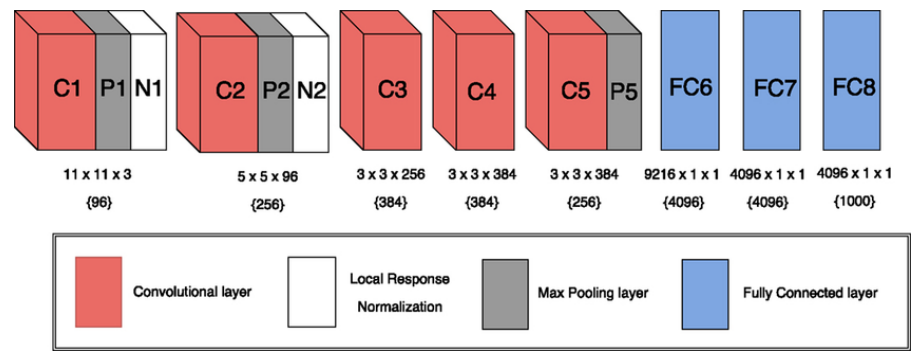

Fig. 5. Alexnet architecture

\section{Googlenet}

This model contains higher number of layer than Alexnet which is 22 layers. It is including 9 inception layers and 13 other layers of convolutional, local response, max pooling and fully connected as depicted in Figure 6 . The input image is fixed to $224 \times 224$ resolution.

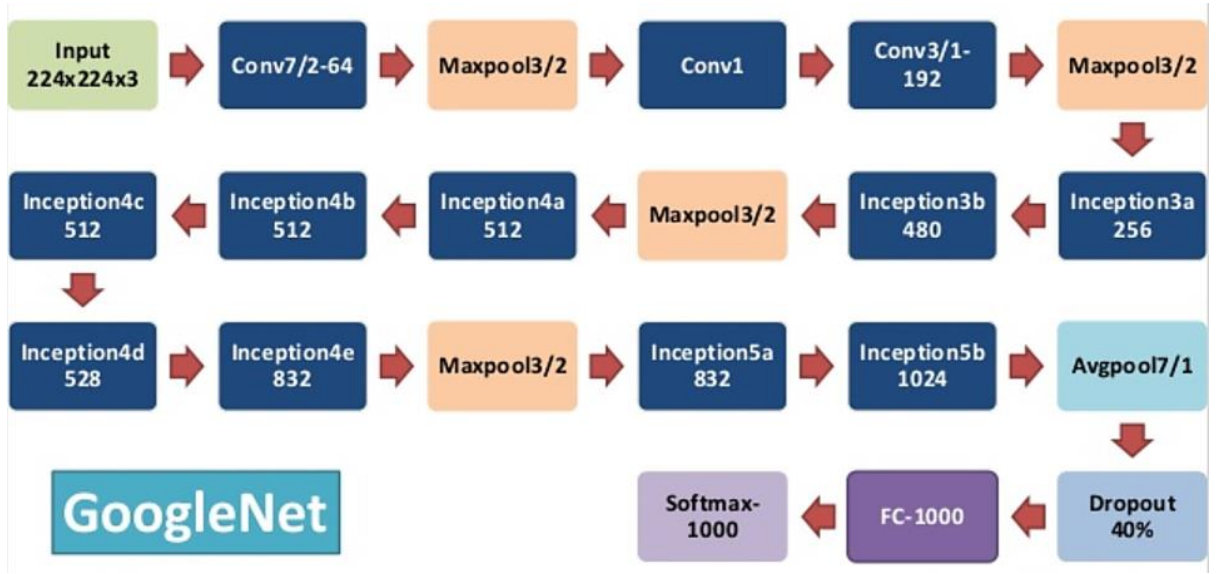

Fig. 6. Googlenet architecture

Inception module is the special feature in this model which convolve data in parallel different size to increase the network depth and width. It also helps to shorten the network's training time. Convolution of $1 \times 1,3 \times 3,5 \times 5$ and max pooling of $3 \times 3$ are done together in parallel way.

\section{VGG-16}

There are 16 layers of convolutional layer, max pooling layer and fully connected layer in VGG-16 model and the input image resolution must be $224 \times 224$. The architecture of VGG-16 can be seen in Figure 7. This model improves its classification accuracy by replacing the large sized convolution filters with small sized filters [22]. 
Max pooling is used to overcome overfitting in this model and reduce the number of learned parameters which will eventually reduce the computational cost.

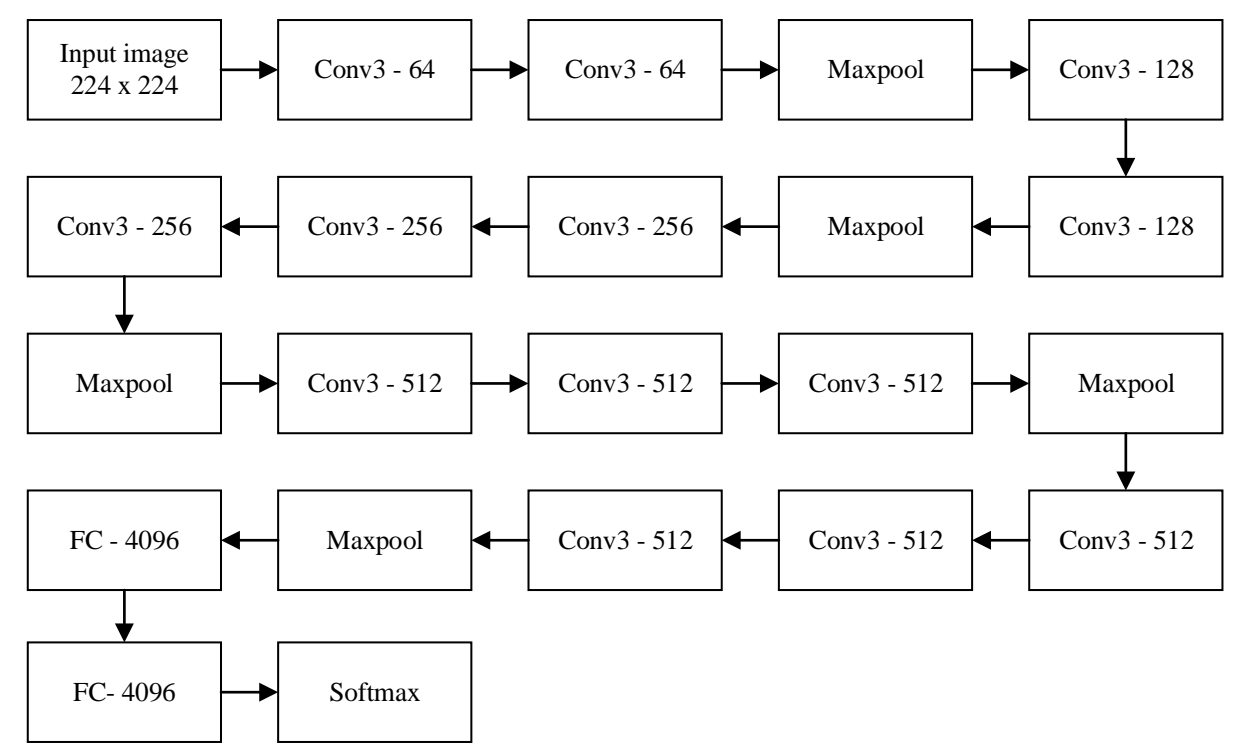

Fig. 7. VGG-16 architecture

4. Mobilenet

Mobilenet has 27 layers in total including 13 depthwise convolution layers, a layer of $3 \times 3$ convolution and $131 \times 1$ convolution layers. Its architecture is as depicted in Figure 8 . It can be seen that there are also average pooling layer, fully connected layer and softmax in Mobilenet structure. Pooling layer is applied to develop downsampling operation to reduce the feature dimension and prevent overfitting [23]. 


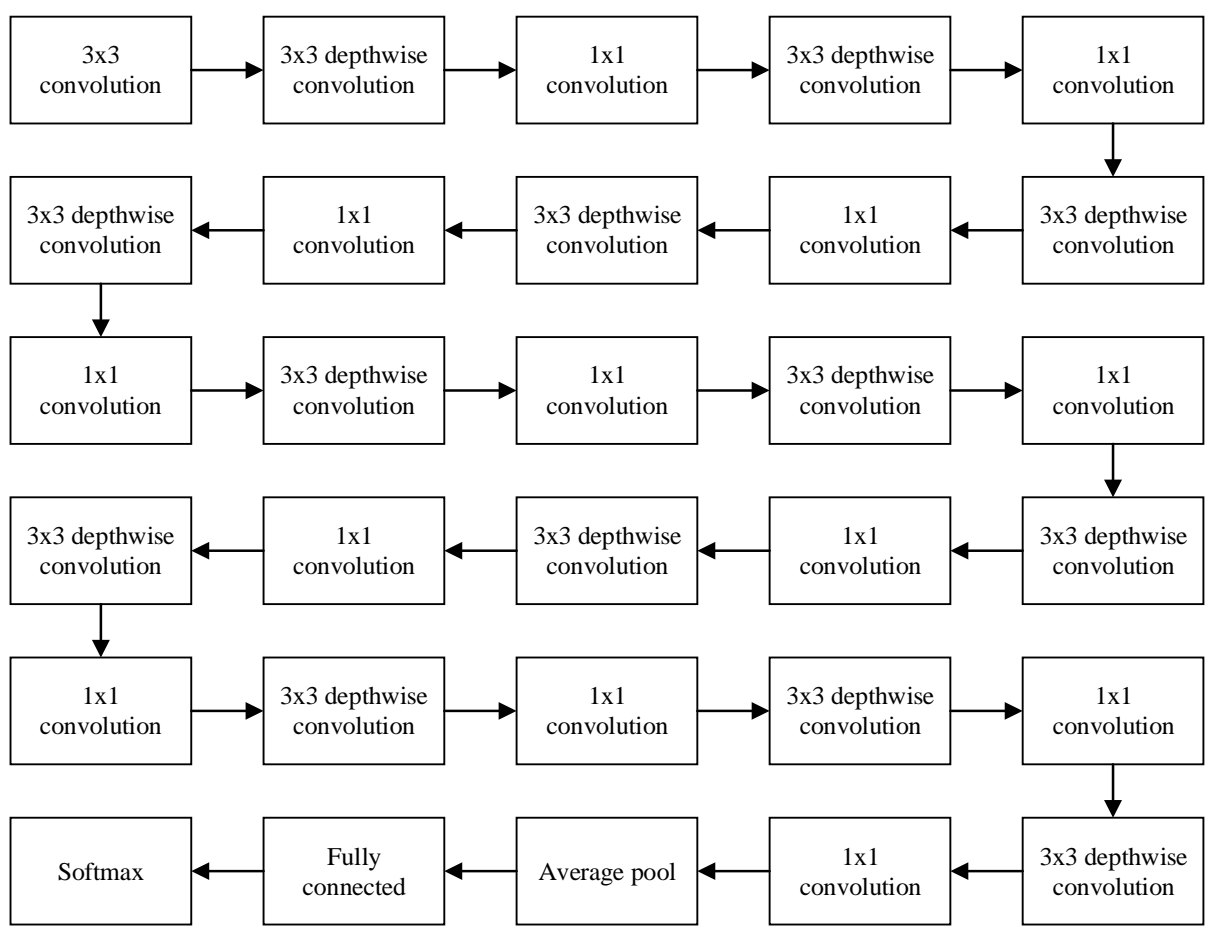

Fig. 8. Mobilenet architecture

Basically, Mobilenet is based on depthwise separable convolution which can be divided into two parts which are depthwise convolution and pointwise convolution. Depthwise convolution is used to apply single filter per each input while pointwise convolution is a simple $1 \times 1$ convolution layer which then used to create a linear combination of depthwise output layer. Depthwise is a step of filtering the input without creating new features while pointwise generates new features. Depthwise separable convolution will help to reduce computational time and size of the model.

\section{Resnet}

Resnet or known as Residual Network is basically based on the residual block with skip connection which add the result from previous layer to the next layer of the model. The reason behind the skip connection is to reduce the training error. In this paper, Resnet of 34 layers is used and the resnet building block is as depicted in Figure 9. Resnet 34 layers contains one max pooling layer and also one average pooling layer at the end of the model. 


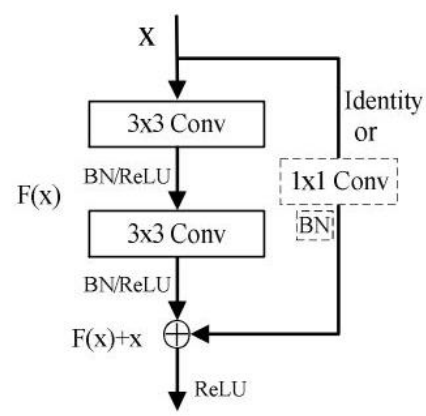

Fig. 9. Resnet building block

Another advantage of applying skip connection is any layer that can affect the performance of the model will be ignored as the it will be skipped by regularization. Other than that, problems involving vanishing or exploding gradient can be prevented.

\section{Densenet}

Densenet uses dense connection between layers through its dense block as shown in Figure 10. It is basically a feed forward connection which the feature maps of all preceding layers are used as input. The importance of this model is to encourage feature reuse and can reduce the number of parameter.

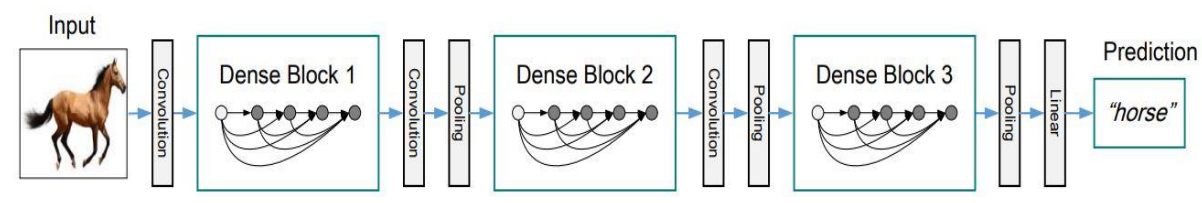

Fig. 10. Densenet connection

\section{$3 \quad$ Results and analysis}

In this section, explanation on the result obtained is made based on three databases. In this paper, CNN is used to classify different types of WBC. Same pre-trained models of Alexnet, Googlenet, VGG-16, Mobilenet, Resnet and Densenet are tested on the three databases. $70 \%$ of images is used for training purposes and another $30 \%$ is used for testing. In training process, the value of epoch and batch size is fixed to 50 and 64 respectively.

\subsection{Kaggle}

Total of 6000 images were tested using six pre-trained models and the result is tabulated in Table 1. 
Table 1. Kaggle result example table

\begin{tabular}{|l|c|c|}
\hline Model & Training Accuracy (\%) & Testing Accuracy (\%) \\
\hline Alexnet & 97.06 & 99.10 \\
\hline Googlenet & 95.26 & 46.57 \\
\hline VGG-16 & 90.91 & 59.55 \\
\hline Mobilenet & 96.29 & 49.83 \\
\hline Resnet & 97.13 & 54.25 \\
\hline Densenet & 86.79 & 62.25 \\
\hline
\end{tabular}

It can be seen from Table 1 that highest training accuracy is achieved by Resnet which is $97.13 \%$ followed by Alexnet and Mobilenet. However, Alexnet obtained the highest testing accuracy by achieving $99.10 \%$. Since Alexnet is the highest for testing, the testing result of each cell type is as shown in Table 2. All types of cell able to achieve high testing accuracy which is more than $98 \%$. Number of misclassified image in Eosinophil category is the highest which is 26. It means that 26 images of Eosinophil are misclassified as other cell type which is Neutrophil. It is followed by Neutrophil, that showed 21 images are misclassified as Eosinophil. These two types of cell are mostly mistaken due to the cell shape, morphological features and the amount of its nucleus which is almost similar and makes it hard to differentiate between those two cells. While as for Lymphocyte and Monocyte, the differences are very clear and easier to classify the cells to their own classes.

Table 2. Testing performance of each cell type (Alexnet)

\begin{tabular}{|l|c|c|c|c|}
\hline \multicolumn{1}{|c|}{ Cell Type } & Eosinophil & Lymphocyte & Monocyte & Neutrophil \\
\hline Testing Accuracy & $98.27 \%$ & $99.80 \%$ & $99.73 \%$ & $98.60 \%$ \\
\hline No. of Correctly Classified Cell & 1474 & 1497 & 1496 & 1479 \\
\hline No. of Misclassified Cell & 26 & 3 & 4 & 21 \\
\hline
\end{tabular}

The breakdown of misclassified image is as shown in Table 3. 26 images of Eosinophil are misclassified as Neutrophil and 20 images of Neutrophil are misclassified as Eosinophil. As for Lymphocyte and Monocyte, the number of misclassified image is low which is 3 and 4 respectively. Most misclassified Neutrophil is mistaken as Eosinophil and vice versa due to its morphological features, number of nucleus in the cytoplasm and their shapes were almost similar to each other. While Lymphocyte and Monocyte has clear morphological features, shape and pattern from one another.

Table 3. Breakdown of misclassified image

\begin{tabular}{|l|l|}
\hline \multicolumn{1}{|c|}{ Cell Type } & \multicolumn{1}{c|}{ Misclassified Images } \\
\hline Eosinophil & $26-$ Neutrophil \\
\hline Neutrophil & $\begin{array}{l}20-\text { Eosinophil } \\
1-\text { Lymphocyte }\end{array}$ \\
\hline Lymphocyte & $\begin{array}{l}2-\text { Eosinophil } \\
1-\text { Monocyte }\end{array}$ \\
\hline Monocyte & $2-$ Neutrophil \\
\hline
\end{tabular}


Average performance for each model is calculated and tabulated in Table 4. Alexnet achieved the highest average performance by obtaining $98.08 \%$ followed by Resnet which the average performance accuracy is $75.69 \%$. The lowest performance accuracy is Googlenet which is $70.92 \%$. Alexnet has showed outstanding performance for Kaggle dataset as other models' performance is in the range of $70 \%-76 \%$ while Alexnet's is $98.08 \%$.

Table 4. Average performance for kaggle dataset

\begin{tabular}{|l|c|}
\hline \multicolumn{1}{|c|}{ Model } & Average Performance (\%) \\
\hline Alexnet & 98.08 \\
\hline Googlenet & 70.92 \\
\hline VGG-16 & 75.23 \\
\hline Mobilenet & 73.06 \\
\hline Resnet & 75.69 \\
\hline Densenet & 74.52 \\
\hline
\end{tabular}

\subsection{IDB-2}

Two classes of data in IDB-2 database which are Lymphoblast and Nonlymphoblast commonly used to detect ALL. Each class contains 130 number of images and the total images in this dataset is 260 images. These images are trained and tested using five different pre-trained models of Alexnet, Googlenet, VGG-16, Mobilenet and Resnet.

The training accuracy and testing accuracy is obtained and kept as shown in Table 5. Highest training accuracy is again achieved by Resnet which is $97.18 \%$. Mobilenet and Alexnet each achieved $96.96 \%$ and $96.15 \%$ training accuracy. The lowest training accuracy is Googlenet. While for testing accuracy, highest is Alexnet which is $96.52 \%$, followed by VGG-16 by obtaining $95.69 \%$ accuracy.

Table 5. Training and testing accuracy

\begin{tabular}{|l|c|c|}
\hline \multicolumn{1}{|c|}{ CNN model } & Training Accuracy (\%) & Testing Accuracy (\%) \\
\hline AlexNet & 96.15 & 96.52 \\
\hline GoogleNet & 88.46 & 83.76 \\
\hline VGG-16 & 91.03 & 95.69 \\
\hline Mobilenet & 96.96 & 72.10 \\
\hline Resnet & 97.18 & 69.99 \\
\hline
\end{tabular}

From the table, it can be said that Alexnet is the best model to classify two types of WBC in IDB database as both its training and testing accuracy is high. However, average performance comparison as shown in Table 6 is made to strengthen the results obtained. Alexnet again showed satisfactory result as Alexnet achieved highest 
average performance accuracy which is $96.34 \%$. The lowest performance accuracy is $83.59 \%$ which has obtained by Resnet. This happens because even though Resnet achieves the highest training accuracy, but its testing performance is the lowest.

Table 6. Average performance for IDB-2 dataset

\begin{tabular}{|l|c|}
\hline \multicolumn{1}{|c|}{ Model } & Average Performance (\%) \\
\hline Alexnet & 96.34 \\
\hline Googlenet & 86.11 \\
\hline VGG-16 & 93.36 \\
\hline Mobilenet & 84.53 \\
\hline Resnet & 83.59 \\
\hline
\end{tabular}

As Alexnet is the best model for classification purposes in IDB-2 database, a detailed testing performance is made as mentioned in Table 7. Performance of testing accuracy for both classes of Lymphoblast and Non-lymphoblast cell is satisfactory which is more than $95 \%$. There is no misclassified image in Lymphoblast class while in Non-lymphoblast class, there is only 4 images that have been misclassified.

Table 7. Accuracy of testing for lymphoblast and non-lymphoblast (Alexnet)

\begin{tabular}{|l|c|c|c|}
\hline \multicolumn{1}{|c|}{ Cell Type } & Lymphoblast (\%) & Non-Lymphoblast $(\boldsymbol{\%})$ & Average Accuracy (\%) \\
\hline Testing Accuracy & 97.74 & 95.29 & 96.52 \\
\hline No. of Misclassified Image & 0 & 4 & - \\
\hline
\end{tabular}

These misclassified images need to be identified as it will give a huge impact to the overall performance. Figure 11 shows some of the misclassified images that often mistaken as the other cell type. These are the images of Lymphoblast and Nonlymphoblast that are misclassified by more than one CNN model. This is probably due to some reasons such as it contains extra noises, confusing shape or color.

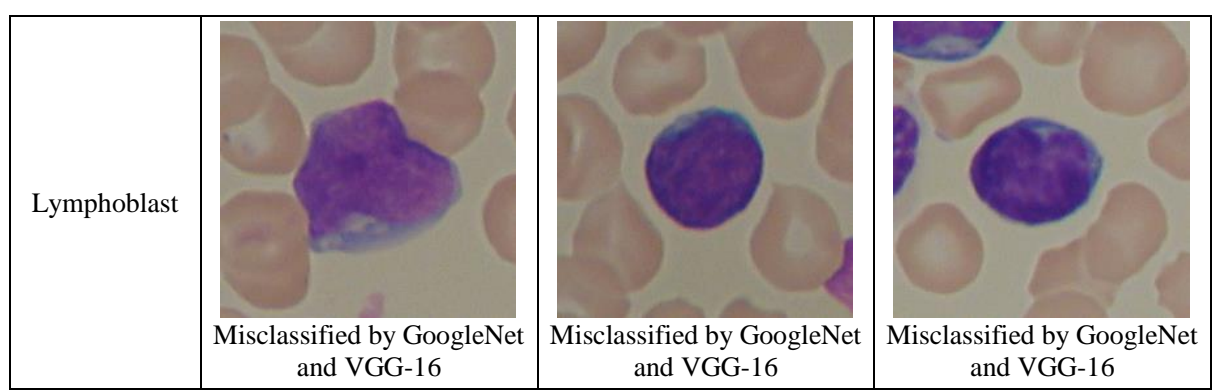




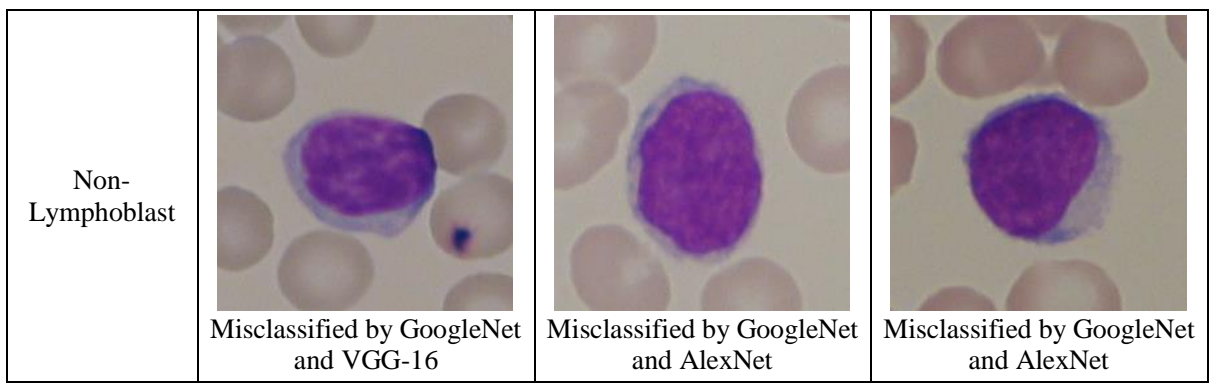

Fig. 11. Problematic images that are often misclassified

\subsection{LISC}

The training and testing result of LISC database is as pictured in Table 8 . Resnet achieved the highest training accuracy which is $97.71 \%$. However, Resnet's testing accuracy is the lowest among other models. While Alexnet is able to achieve highest training accuracy which is $88.21 \%$ with training accuracy of $80.82 \%$.

Table 8. Training accuracy of LISC database

\begin{tabular}{|l|c|c|}
\hline \multicolumn{1}{|c|}{ Model } & Training Accuracy (\%) & Testing Accuracy (\%) \\
\hline AlexNet & 80.82 & 88.21 \\
\hline GoogleNet & 73.97 & 75.46 \\
\hline VGG-16 & 76.71 & 80.58 \\
\hline MobileNet & 94.13 & 57.78 \\
\hline ResNet & 97.71 & 53.70 \\
\hline
\end{tabular}

Average performance for each model is calculated as shown in Table 9. It can be seen that the best model to classify 5 types of WBC in LISC dataset is achieved by Alexnet which is $84.52 \%$ accuracy. The lowest average performance is done by Googlenet by obtaining $74.72 \%$ of accuracy.

Table 9. Average performance for LISC dataset

\begin{tabular}{|l|c|}
\hline \multicolumn{1}{|c|}{ Model } & Average Performance (\%) \\
\hline Alexnet & 84.52 \\
\hline Googlenet & 74.72 \\
\hline VGG-16 & 78.65 \\
\hline Mobilenet & 75.96 \\
\hline Resnet & 75.71 \\
\hline
\end{tabular}

Testing performance of each cell type for Alexnet is done as Alexnet achieves highest performance for LISC dataset. It is as depicted in Table 10. Basophil, Neutrophil, Lymphocyte and Monocyte achieve satisfactory testing performance. However, testing performance of Eosinophil is the lowest which is $75.37 \%$. Next, number of 
misclassified image is calculated and there is no misclassified image in Neutrophil category. It means that all Neutrophil images are correctly classified. Overall, total number of misclassified image of 5 types of WBC is 16 images which can be considered as low and acceptable.

Table 10. Alexnet's testing accuracy of LISC database

\begin{tabular}{|l|c|c|c|c|c|c|}
\hline \multicolumn{1}{|c|}{ Cell Type } & Basophils & Eosinophils & Neutrophils & Lymphocyte & Monocyte & Average Accuracy (\%) \\
\hline $\begin{array}{l}\text { Testing Accu- } \\
\text { racy (\%) }\end{array}$ & 91.70 & 75.37 & 98.10 & 95.11 & 80.76 & 88.21 \\
\hline $\begin{array}{l}\text { No. of Misclas- } \\
\text { sified Images }\end{array}$ & 4 & 6 & 0 & 1 & 5 & Total = 16 \\
\hline
\end{tabular}

Some of the images that have been misclassified is as pictured in Table 11. These images have been misclassified by all pre-trained models. This is most probably due to its acquisition condition, different lighting and coloration. Other than that, the color intensity of the image is not standardized. Hence, it is misclassified by all pre-trained models.

Table 11. Sample of misclassified images

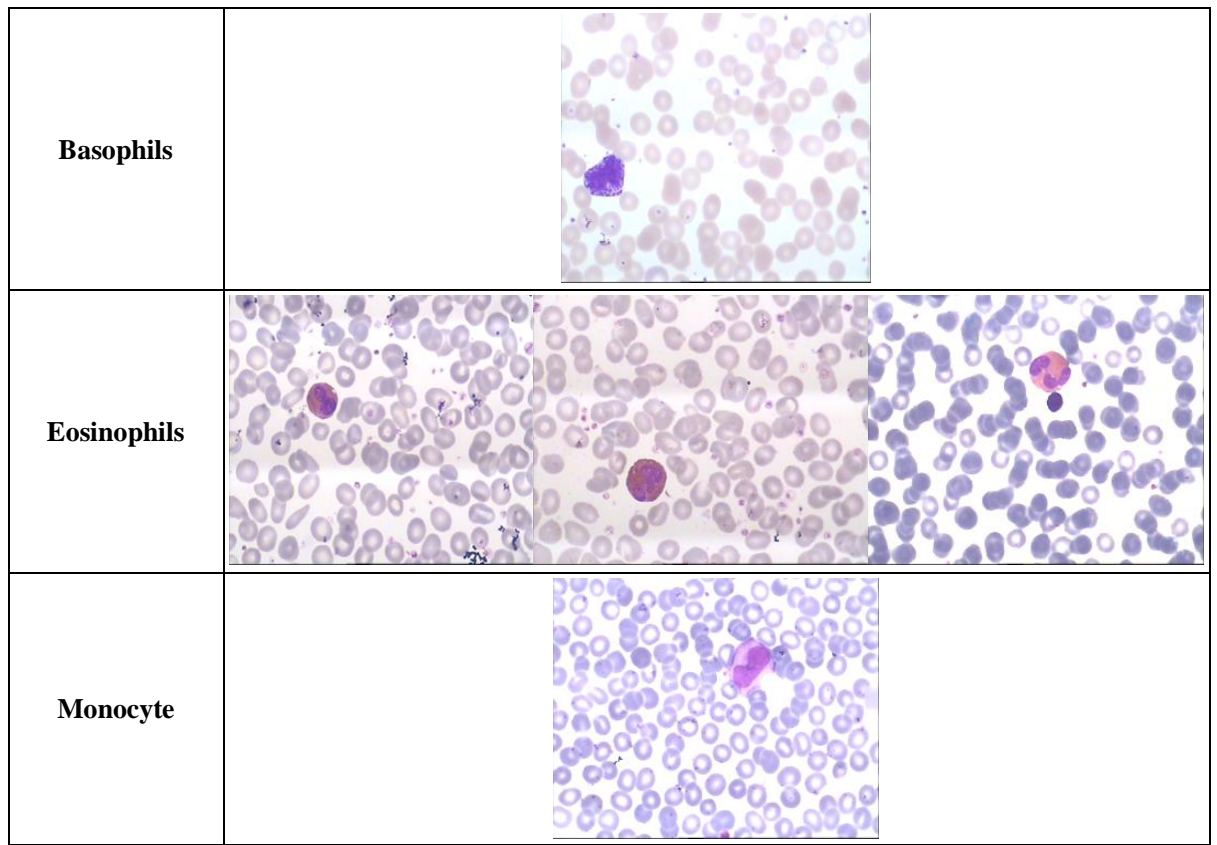

\section{$4 \quad$ Conclusion and future works}

In this paper, several $\mathrm{CNN}$ pre-trained models were tested on three databases. The purpose of this project is to classify WBC as these databases contain different WBC 
type. The platform used is Matlab R2020a and deep learning toolbox is included. Epoch value and batch size is fixed for all models which is 50 and 64 respectively.

Firstly, pre-trained models of Alexnet, Googlenet, VGG-16, Mobilenet, Resnet and Densenet is applied on Kaggle dataset to classify 4 types of WBC which is Eosinophil, Neutrophil, Lymphocyte and Monocyte. There are 1500 images in each class which will total up to 6000 images in Kaggle dataset. These images were trained and tested, and the comparison between each model is observed and recorded. In training process, Resnet achieved the highest accuracy of $97.13 \%$ followed by Alexnet which is $97.06 \%$. However, as for testing accuracy, Alexnet is able to obtain the highest accuracy by achieving $99.10 \%$. Average performance is calculated and based on the findings, Alexnet is the best model to classify WBC types in Kaggle dataset as it achieved $98.08 \%$ performance accuracy. It is definitely higher than other models.

Next, Alexnet, Googlenet, VGG-16, Mobilenet and Resnet is used to classify two types of cell in IDB-2 database which is Lymphoblast and Non-lymphoblast. Each class of data contains 130 number of images and total image in the database is 260 images. The comparison of the performance is made and it is found that the training accuracy of Resnet is the highest which is $97.18 \%$ while second highest is Alexnet by achieving $96.15 \%$ accuracy. As for testing performance, $96.52 \%$ accuracy is the highest which is achieved by Alexnet and followed by VGG-16 which achieved $95.69 \%$ testing accuracy. In order to find the best model for IDB-2 database, average performance is made and the highest performance accuracy is achieved by Alexnet which is 96.34\%. Moreover, Alexnet is able to classify all Lymphoblast image precisely.

Lastly, in LISC dataset, there are 5 classes of data which are Eosinophil, Neutrophil, Basophil, Monocyte and Lymphocyte. Each class consists of 39, 50, 53, 52 and 48 images respectively and total image in LISC dataset is 242 images. Resnet is able to achieve the highest training accuracy which is $97.71 \%$. However, its testing accuracy is $53.70 \%$ accuracy which is the lowest among other model. The highest testing accuracy is obtained by Alexnet by achieving accuracy of $88.21 \%$. Average performance of each model is calculated and it is found that Alexnet is the best model to classify WBC types in LISC dataset as it achieved $84.52 \%$ of accuracy. Googlenet's average performance is the lowest which is $74.72 \%$. Number of misclassified images by Alexnet is 16 images out of 242 images which is considered low.

Overall, as discussed in the previous section, Alexnet is the best model to classify WBC types in all three databases of Kaggle, IDB-2 and LISC. It contains the lowest number of layers compared to other models that have been tested.

In future, the project's finding is expected to be improved by adding the comparison between several optimizers such as Adam, RMSprop and Stochastic Gradient Descent with Momentum (SGDM). Other than that, fine tuning and developing basic own model is also to be made for the results improvement. Lastly, the dataset should be increase to strengthen the findings. 


\section{Acknowledgement}

This research is supported by Ministry of Higher Education (MOHE) through Fundamental Research Grant Scheme (FRGS) (FRGS/1/2019/TK04/UTHM/02/6) and Universiti Tun Hussein Onn Malaysia (UTHM) through Postgraduate Research Grant GPPS (vot H400).

\section{$6 \quad$ References}

[1] Kutlu, H., E. Avci, and F. Özyurt, White blood cells detection and classification based on regional convolutional neural networks. Medical hypotheses, 2020. 135: p. 109472. https://doi.org/10.1016/j.mehy.2019.109472

[2] Macawile, M.J., et al. White blood cell classification and counting using convolutional neural network. in 2018 3rd International conference on control and robotics engineering (ICCRE). 2018. IEEE. https://doi.org/10.1109/iccre.2018.8376476

[3] Hegde, R.B., et al., Comparison of traditional image processing and deep learning approaches for classification of white blood cells in peripheral blood smear images. Biocybernetics and Biomedical Engineering, 2019. 39(2): p. 382-392. https://doi.org/10.1016/j. bbe.2019.01.005

[4] Zheng, X., et al., Fast and robust segmentation of white blood cell images by selfsupervised learning. Micron, 2018. 107: p. 55-71. https://doi.org/10.1016/j.micron.2018.01 .010

[5] Safuan, S.N.M., M.R.M. Tomari, and W.N.W. Zakaria, White blood cell (WBC) counting analysis in blood smear images using various color segmentation methods. Measurement, 2018. 116: p. 543-555. https://doi.org/10.1016/j.measurement.2017.11.002

[6] Novoselnik, F., et al. Automatic white blood cell detection and identification using convolutional neural network. in 2018 International Conference on Smart Systems and Technologies (SST). 2018. IEEE. https://doi.org/10.1109/sst.2018.8564625

[7] Banik, P.P., R. Saha, and K.-D. Kim, An automatic nucleus segmentation and CNN model based classification method of white blood cell. Expert Systems with Applications, 2020. 149: p. 113211. https://doi.org/10.1016/j.eswa.2020.113211

[8] Thanh, T., et al., Leukemia blood cell image classification using convolutional neural network. International Journal of Computer Theory and Engineering, 2018. 10(2): p. 54-58.

[9] Habibzadeh, M., et al. Automatic white blood cell classification using pre-trained deep learning models: ResNet and Inception. in Tenth international conference on machine vision (ICMV 2017). 2018. International Society for Optics and Photonics. https://doi.org/10. $\underline{1117 / 12.2311282}$

[10] Yamashita, R., et al., Convolutional neural networks: an overview and application in radiology. Insights into imaging, 2018. 9(4): p. 611-629.

[11] Baheti, B., S. Gajre, and S. Talbar. Detection of distracted driver using convolutional neural network. in Proceedings of the IEEE conference on computer vision and pattern recognition workshops. 2018. https://doi.org/10.1109/cvprw.2018.00150

[12] Acharya, U.R., et al., A deep convolutional neural network model to classify heartbeats. Computers in biology and medicine, 2017. 89: p. 389-396. https://doi.org/10.1016/j.comp biomed.2017.08.022

[13] Khan, H.A., et al., Brain tumor classification in MRI image using convolutional neural network. Math. Biosci. Eng, 2020. 17: p. 6203. 
[14] Ayesha Samreen, A.M.T., Yasa Vishwanath Reddy, Sathish P, Brain Tumor Detection by Using Convolution Neural Network. International Journal of Online and Biomedical Engineering, 2020. 16(13): p. 58. https://doi.org/10.3991/ijoe.v16i13.18545

[15] Lee, S.-G., et al., Variations of AlexNet and GoogLeNet to improve Korean character recognition performance. Journal of information processing systems, 2018. 14(1): p. 205217.

[16] Huang, F., et al. Chinese herbal medicine leaves classification based on improved AlexNet convolutional neural network. in 2019 IEEE 4th Advanced Information Technology, Electronic and Automation Control Conference (IAEAC). 2019. IEEE. https://doi.org/10.1109/ iaeac47372.2019.8997578

[17] Sam, S.M., et al., Offline signature verification using deep learning convolutional neural network (CNN) architectures GoogLeNet Inception-v1 and Inception-v3. Procedia Computer Science, 2019. 161: p. 475-483. https://doi.org/10.1016/j.procs.2019.11.147

[18] Fang, T. A novel computer-aided lung cancer detection method based on transfer learning from GoogLeNet and median intensity projections. in 2018 IEEE international conference on computer and communication engineering technology (CCET). 2018. IEEE. https://doi. org/10.1109/ccet.2018.8542189

[19] Ahmed Elnakib, H.M.A., Fatma E.Z. Abou-Chadi, Early Lung Cancer Detection using Deep Learning Optimization. International Journal of Online and Biomedical Engineering, 2020. 16(6): p. 82. https://doi.org/10.3991/ijoe.v16i06.13657

[20] Guo, T., et al. Simple convolutional neural network on image classification. in 2017 IEEE 2nd International Conference on Big Data Analysis (ICBDA). 2017. IEEE. https://doi.org/ 10.1109/icbda.2017.8078730

[21] Samir Abou El-Seoud, M.H.S., Gerard McKee, Detection and Classification of White Blood Cells through Deep Learning Techniques. international Journal of Online and Biomedical Engineering, 2020. 16(15): p. 94. https://doi.org/10.3991/ijoe.v16i15.15481

[22] Xu, G., et al., A deep transfer convolutional neural network framework for EEG signal classification. IEEE Access, 2019. 7: p. 112767-112776. https://doi.org/10.1109/access.20 $\underline{19.2930958}$

[23] Pan, H., et al., A new image recognition and classification method combining transfer learning algorithm and mobilenet model for welding defects. IEEE Access, 2020. 8: p. 119951-119960. https://doi.org/10.1109/access.2020.3005450

\section{$7 \quad$ Authors}

Syadia Nabilah Mohd Safuan is with Faculty of Electronic and Electrical Engineering, Universiti Tun Hussein Onn Malaysia, Batu Pahat, Malaysia.

Mohd Razali Md Tomari is with Faculty of Electronic and Electrical Engineering, Universiti Tun Hussein Onn Malaysia, Batu Pahat, Malaysia, and he is a principle researcher in Research Centre for Applied Electromagnetics, Johor, Malaysia.

Wan Nurshazwani Wan Zakaria is with Faculty of Electronic and Electrical Engineering, Universiti Tun Hussein Onn Malaysia, Batu Pahat, Malaysia.

Article submitted 2021-10-05. Resubmitted 2021-11-16. Final acceptance 2021-11-18. Final version published as submitted by the authors. 